\title{
Carbon-Supported PtNi Nanocrystals for Alkaline Oxygen Reduction and Evolution Reactions; Electrochemical Activity and Durability upon Accelerated Stress Tests
}

\author{
Victor Shokhen ${ }^{1-2}$, Melina Zysler ${ }^{2}$, Meital Shviro³, David Zitoun*2, Marian Chatenet*1 \\ 1- Univ. Grenoble Alpes, Univ. Savoie Mont Blanc, CNRS, Grenoble INP (Institute of Engineering Univ. \\ Grenoble Alpes), LEPMI, 38000 Grenoble, France \\ 2- Department of Chemistry and Bar-Ilan Institute for Technology and Advanced Materials (BINA), \\ Bar-llan University, Ramat Gan 5290002, Israel \\ 3- Institute of Electrochemical and Climate Research IEK-14, Forschungszentrum Jülich GmbH, 52425 \\ Jülich, Germany \\ Corresponding author: \\ *E-mail: David.Zitoun@biu.ac.il \\ *E-mail: Marian.Chatenet@grenoble-inp.fr
}




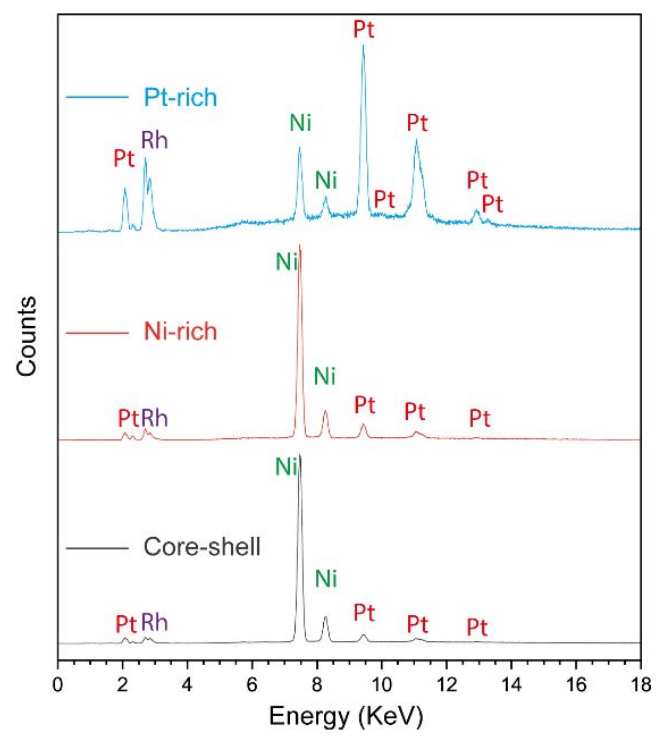

Figure S1. XRF spectra for Pt-Ni pellet samples (10wt\% sample and 90wt\% Dextran per pellet). Five spots of $100 \mu \mathrm{m}$ diameter were measured for $120 \mathrm{~s}$ per sample, the acquisition being made at $50 \mathrm{kV}$. Bottom: Core-shell, middle: Ni-rich, top: Pt-rich. The peak at ca. $2.8 \mathrm{kV}$ corresponds to Rh (the tube source).
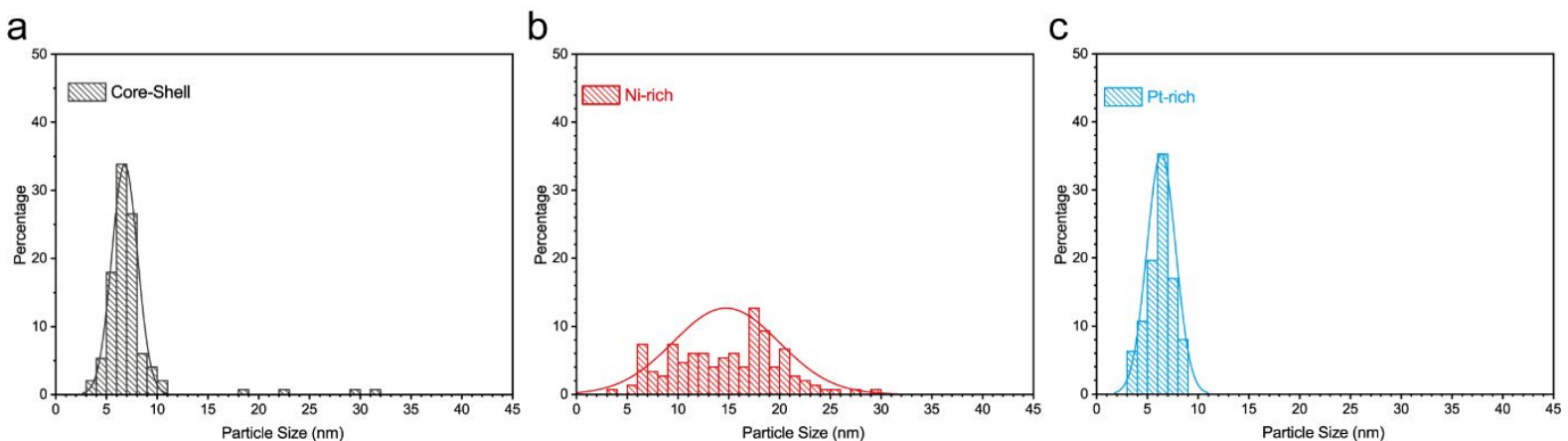

Figure S2. Particle size distribution (PSD) histograms of the three PtNi NPs types obtained upon their synthesis (initial state); a) Pt@Ni Core-shell, b) Ni-rich PtNi, and c) Pt-rich PtNi. 

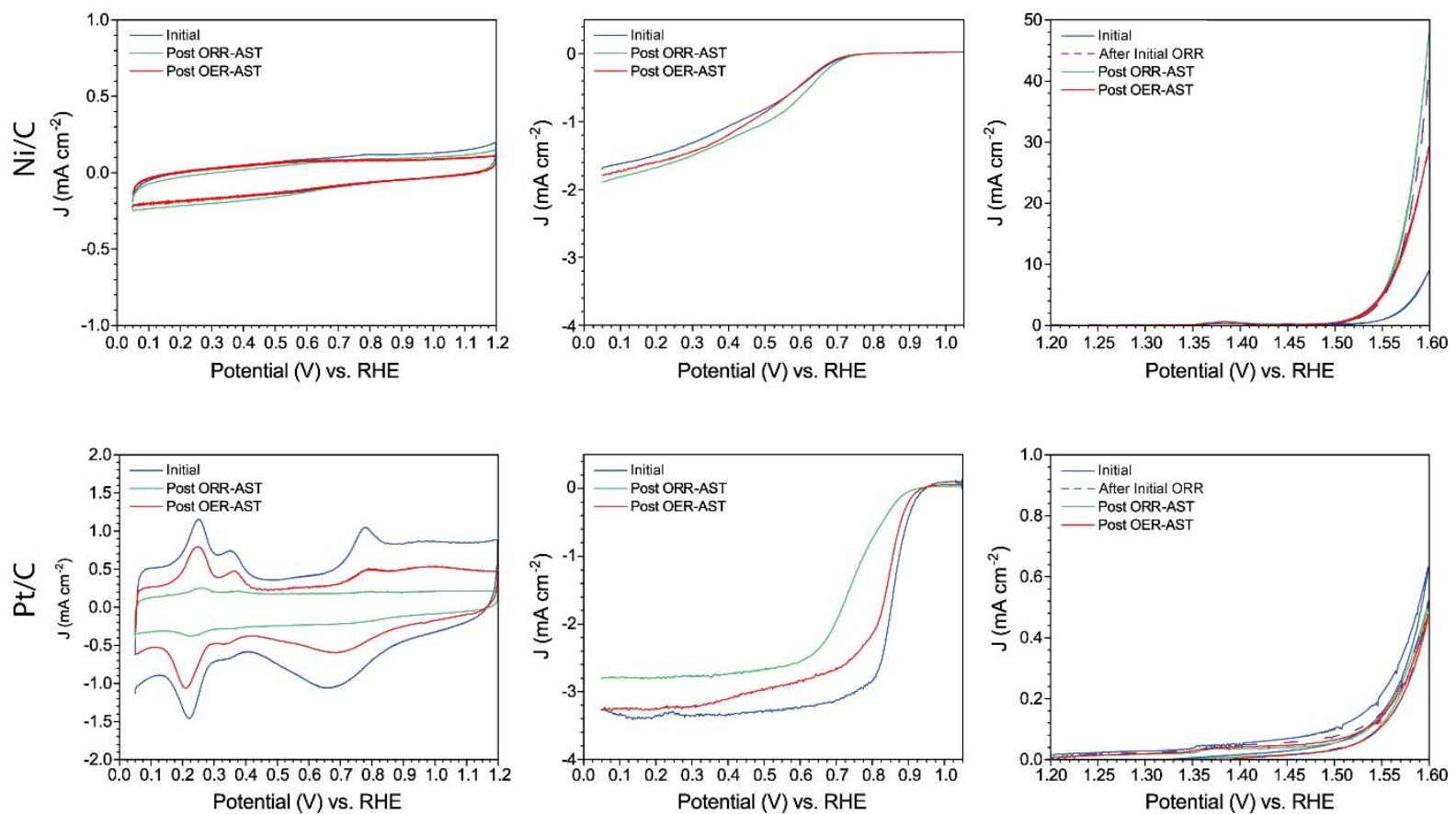

Figure S3. Evolution of the electrochemical performance of the commercial catalysts $\mathrm{Ni} / \mathrm{C}$ and $\mathrm{Pt} / \mathrm{C}$ post-OER or ORR AST. Oxygen evolution reaction (OER) curve (a), Oxygen reduction reaction (ORR) curve (b), Cyclic voltammetry (CV) curve (c).
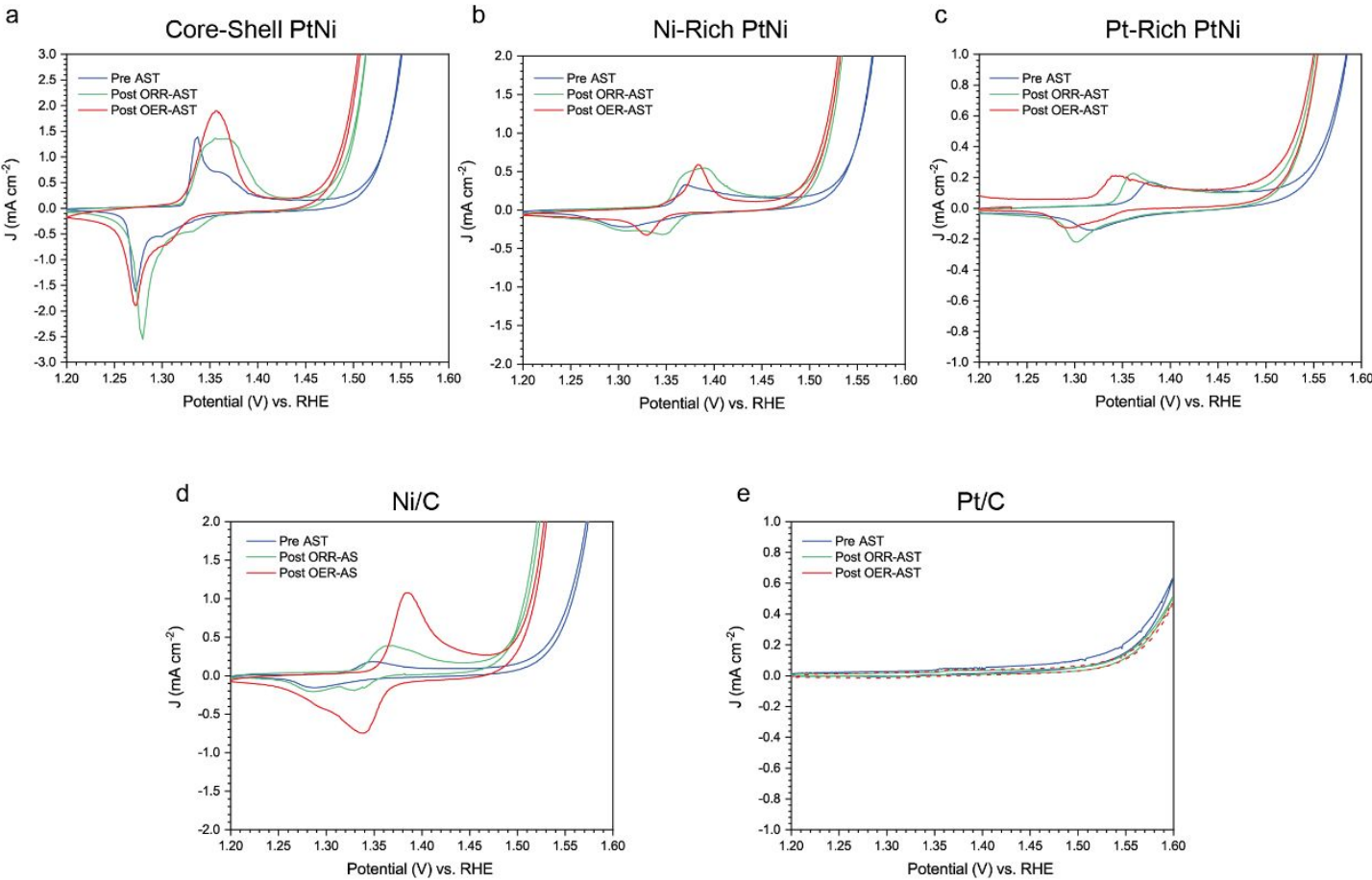

Figure S4. $\mathrm{Ni}^{2+} / \mathrm{Ni}^{3+}$ redox region of the catalyst in initial pre AST state and post AST. 

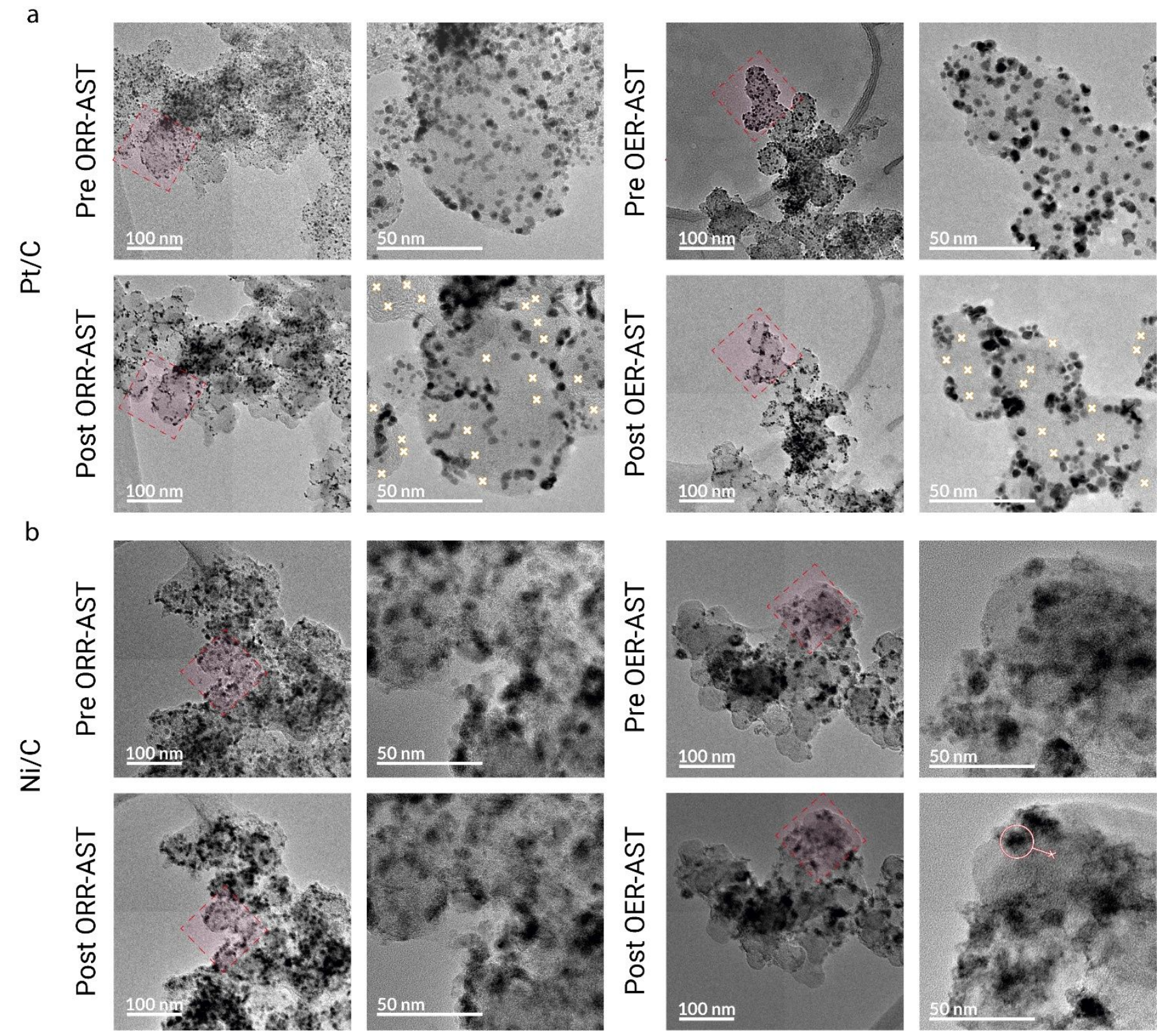

Figure S5. Identical location transmission electron microscope (IL-TEM) micrograph of the commercial particles; Pt/C and Ni/C samples, pre and post ORR-AST or OER-AST. Orange-white " $X$ " are related to some missing particles post-AST (but this marker is non-comprehensive). The red circle defines a particle "drift". 
a

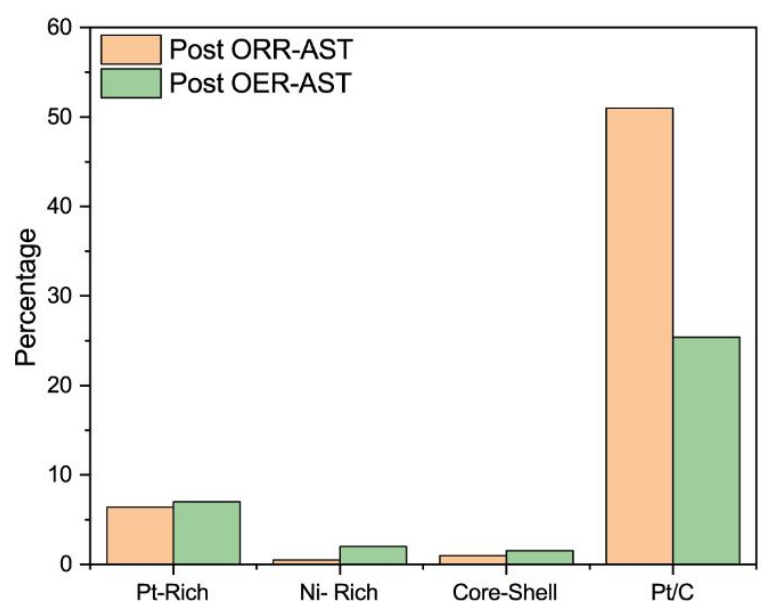

b

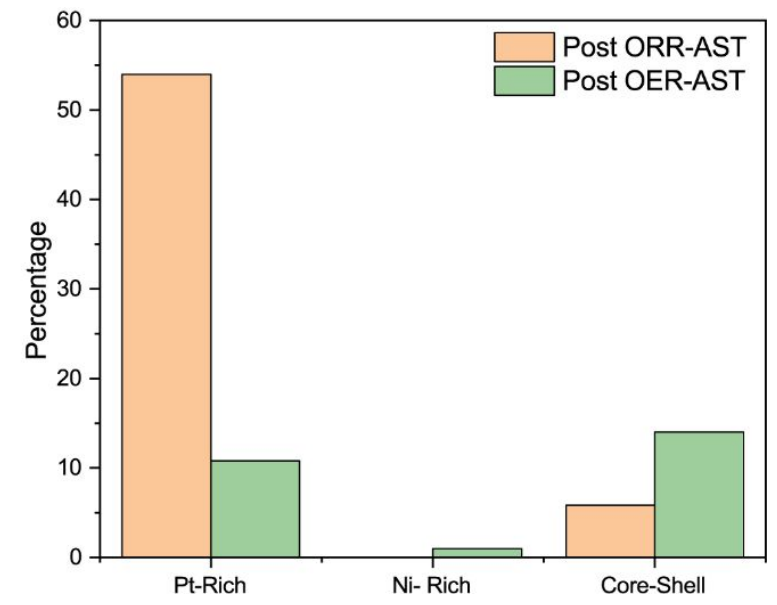

Figure S6. histograms of the percentage of lost particles after AST (a), and percentage of shapechanged PtNi particles after the AST process (b).

a

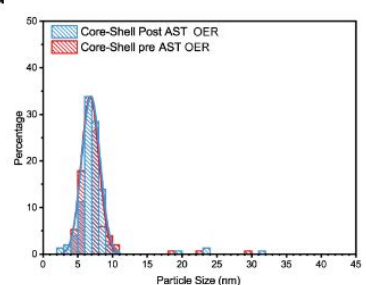

b
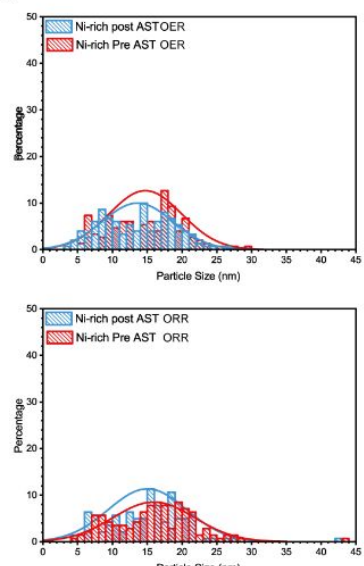
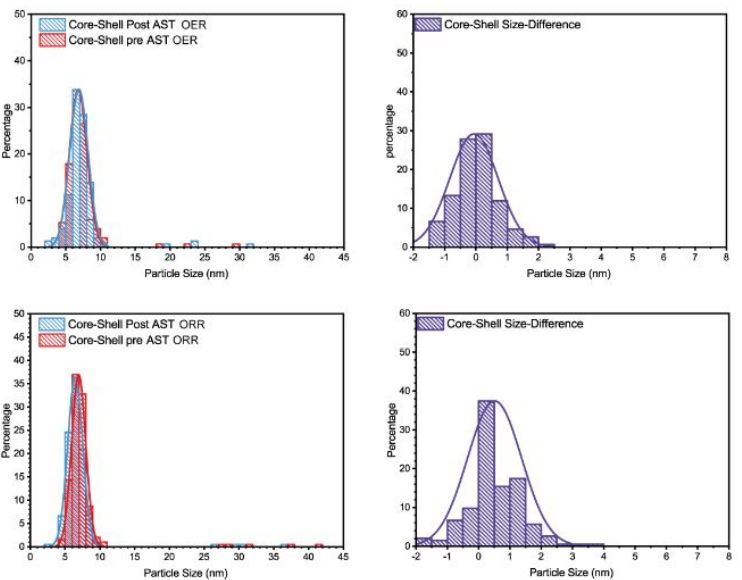

C
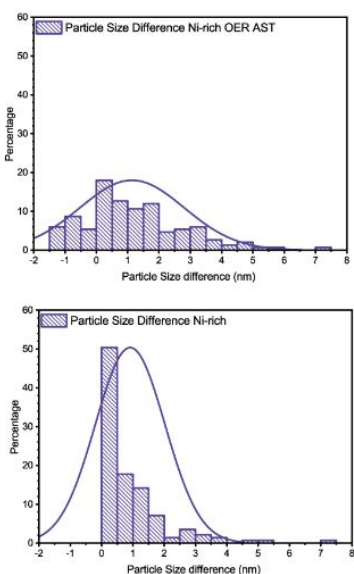
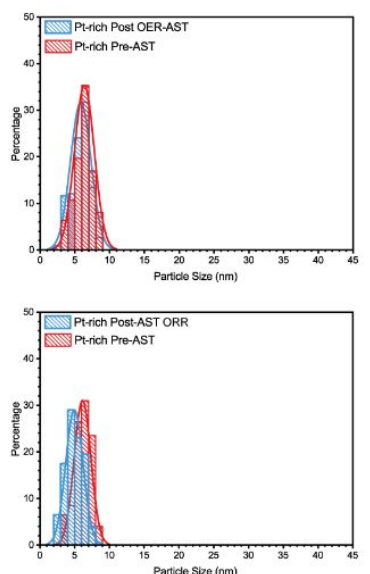
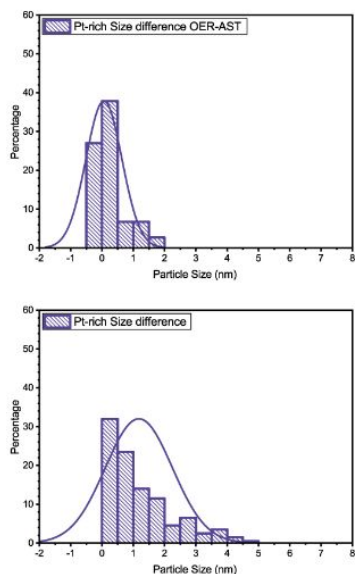

Figure S7. PSD of the PtNi sample before (Red histogram) and post of each AST type (Blue histogram) with the size difference (Purple histogram) negative number mean an increase in size, while positive mean shrinking in size. 

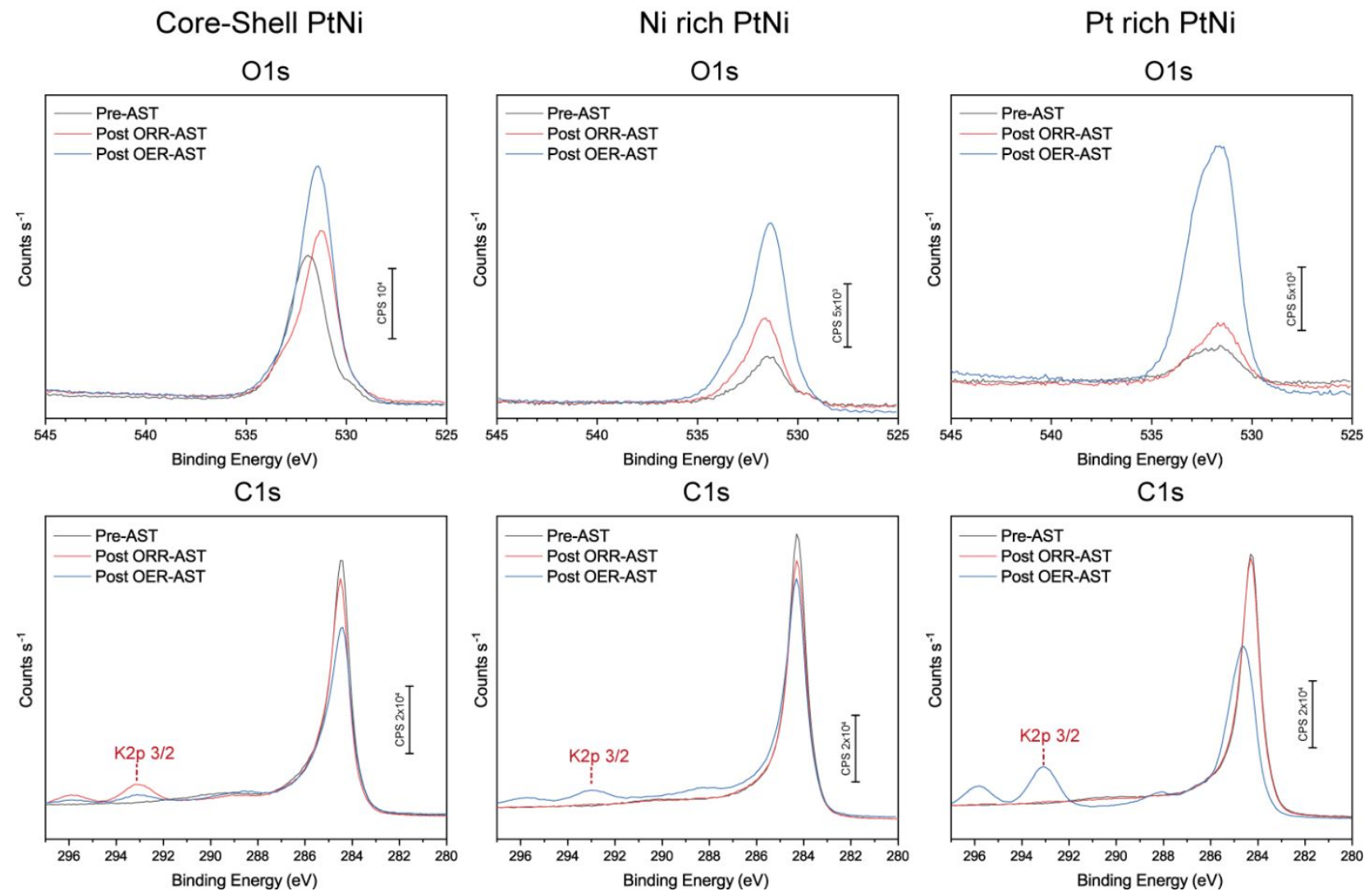

Figure S8. XPS plots of C1s and O1s, pre and post AST of the PtNi samples corresponding to Fig 8.

C1s

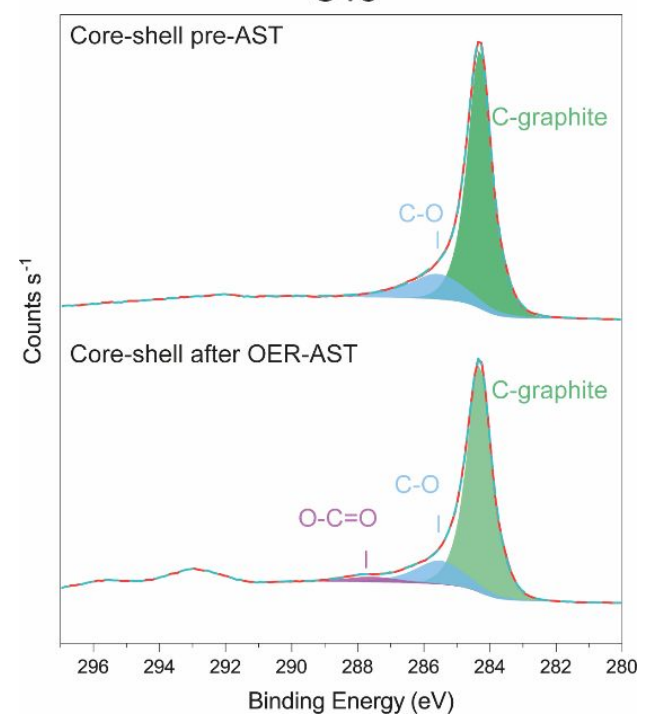

Figure S9. XPS plot of C1s with a peak fitting of carbon, pre-AST and post OER-AST of the PtNi coreshell. 